\title{
Factor XII (Hageman factor) is over-expressed in brain metastatic breast cancer.
}

Shahan Mamoor

East Islip, NY USA ${ }^{1}$

1'shahanmamoor@gmail.com

Brain metastases are a clinical problem in patients with breast cancer ${ }^{1-3}$. We mined published microarray data $^{4,5}$ to discover genes associated with brain metastasis in patients with brain metastatic breast cancer. We found that the gene encoding the coagulation factor XII (FXII), also known as Hageman factor, F12, was among the genes most differentially expressed in the brain metastases of patients with brain metastatic breast cancer. FXII may be of relevance to the biology underlying metastasis to the brain, and it may be of relevance as a potential therapeutic target in patients with intractable disease.

Keywords: breast cancer, brain metastases, central nervous system metastases, F12, factor XII, systems biology of breast cancer, targeted therapeutics in breast cancer. 
One report described a 34\% incidence of central nervous system metastases in trastuzumabtreated patients with breast cancer ${ }^{2}$. This alarmingly high frequency of CNS metastasis events demands an enhanced understanding of the transcriptional makeup of brain metastatic tissues to support identification of therapeutic targets. We performed a global comparative analysis of transcription in the brain metastases of patients with brain metastatic breast cancer ${ }^{4,5}$. We discovered significant differential and over-expression of factor XII, a coagulation factor with described expression in the brain 6 , in brain metastases from patients with brain metastatic breast cancer.

\section{Methods}

We used datasets GSE1259894 and GSE526045 for this global differential gene expression analysis of brain metastatic breast cancer in conjunction with GEO2R. GSE125989 was generated using Affymetrix Human Genome U133A 2.0 Array technology with $n=16$ primary tumors from patients with breast cancer and $n=16$ brain metastases from patients with brain metastatic breast cancer. GSE52604 was generated using Whole Human Genome Microarray 4x44K G4112F technology with $n=10$ normal breast tissues and $\mathrm{n}=35$ brain metastases from patients with breast cancer, analysis performed using platform GPL6480. The Benjamini and Hochberg method of $p$-value adjustment was used for ranking of differential expression but raw $p$-values were used to assess statistical significance of global differential expression. Log-transformation of data was auto-detected, and the NCBI generated category of platform annotation was used. A statistical test was performed to evaluate whether F12 gene expression was significantly between primary breast tumors and brain metastases in humans with breast cancer using a two-tailed, unpaired t-test with Welch's correction. We used PRISM for all statistical analyses of differential gene expression in human breast cancer (Version 8.4.0)(455).

\section{Results}

We performed global comparative transcriptome analysis of the primary and metastatic tumor tissues of patients with brain metastatic breast cancer using published microarray data ${ }^{4,5}$ to describe the transcriptional landscape of brain metastasis in human breast cancer in an unbiased fashion and for the discovery of novel therapeutic targets.

\section{F12 is differentially expressed in the brain metastases of patients with brain metastatic breast} cancer.

We identified the gene encoding factor XII, F12, as among the genes whose expression was most significantly different in the brain metastases of patients with brain metastatic breast cancer ${ }^{4}$ (Table 1). When sorting each of the genes expressed in brain metastases based on significance of difference as compared to the primary tumors of patients with brain metastatic breast cancer, F12 ranked 378 out of 22297 total transcripts (Table 1), equating to $98.3 \%$ differential expression. Differential expression of F12 in the brain metastases of patients with brain metastatic breast cancer was statistically significant (Table 1; $p=5.04 \mathrm{E}-03$ ).

Cross-validation of differentially expressed genes across datasets in cancer can be difficult and more challenging than when cross-validating across organs and sorted cell populations due to higher levels of heterogeneity in tumors, and differing methods of tumor sampling between laboratories. Thus, we asked whether we could validate differential expression of F12 when comparing brain metastases from patients with breast cancer to normal breast tissue as opposed to primary tumors of the breast. Comparison of global gene expression profiles of 10 normal breast tissues to 35 brain metastases 5 revealed that F12 was among the genes whose expression was most significantly different 
transcriptome-wide between the breast and brain metastases in patients with breast cancer (Table 2). When sorting each of the genes expressed in brain metastases based on significance of difference in expression between brain metastases and normal breast tissues, F12 ranked 7919 out of 41093 total transcripts (Table 2), equating to $80.7 \%$ differential expression. Differential expression of F12 in brain metastases from patients with brain metastatic breast cancer in this dataset was statistically significant (Table $2 ; p=1.75 \mathrm{E}-03$ ).

\section{F12 is expressed at significantly higher levels in the brain metastases of patients with brain metastatic breast cancer.}

We obtained exact mRNA expression levels for F12, in primary tumors of the breast and in brain metastasis of patients with brain metastatic breast cancer to determine magnitude and direction of change in F12 expression in brain metastatic tissues. F12 was expressed at higher levels in the brain metastases of patients with breast cancer as compared to primary tumors of the breast, and this difference was statistically significant (Figure $1 ; p=0.0012)$. We calc expression when comparing brain metastatic tissues to primary tumors of the breast in patients with breast cancer (Table 1).

Thus, by mining published microarray data 4,5 in an unbiased and systematic fashion, we identified coagulation factor XII, F12, as among the genes whose expression was most different in the brain metastases of patients with breast cancer both when compared to primary tumors of the breast and to the normal breast; F12 was expressed at significantly higher levels in brain metastases as compared to primary tumors of the breast.

\section{Discussion}

Coagulation, the activation of platelets by soluble factors to form solid matter known as clots, transpires through a cascade of reactions with schematic similarity to the complement cascade, and can proceed through the extrinsic or intrinsic pathways 7,8 . Factor XII is involved in the intrinsic pathway of coagulation ${ }^{8}$. Factor XII (FXII) is the zymogen, or inactive form of factor XIIa (FXIIa), which once activated initiates a cascade resulting in activation of factor XI, then factor IX and factor X, and finally to thrombin, the central effector necessary for coagulation (e.g., generation of a fibrin network from fibrinogen $)^{8}$. Mice null for factor XII are unable to form and stabilize thrombus but with no excessive bleeding spontaneously or in response to excessive injury, indicating that FXII is critical for the formation of thrombus ${ }^{9}$. Activation of FXII can occur through plasma kallikrein or through auto activation in response to a number of different stimuli ${ }^{10}$. Aside from its role in coagulation, FXII can support inflammatory responses through bradykinin through kininogen and plasma kallikrein in a system known as the kallikrein kinin system ${ }^{10}$. Factor XII has been reported as up-regulated in the peritoneum of patients with epithelial ovarian cancer ${ }^{11}$. In a breast cancer model using injection of Mtln3 rat mammary carcinoma cells intravenously into Fischer 344 rats, factors II, VII, IX and X increased the number of metastases at the pulmonary surface, leading authors to suggest that factors II, IX and X possessed an ability or component which could support metastasis ${ }^{12}$.

We report here that coagulation factor XII, also known as Hageman factor, is among the genes whose expression is most different in the brain metastases of patients with brain metastatic brain cancer. FXII may be of relevance to the biology underlying metastasis to the brain in patients with brain metastatic breast cancer, a disease with limited treatment options. 


\section{References}

1. Lin, N.U., Amiri-Kordestani, L., Palmieri, D., Liewehr, D.J. and Steeg, P.S., 2013. CNS metastases in breast cancer: old challenge, new frontiers.

2. Bendell, J.C., Domchek, S.M., Burstein, H.J., Harris, L., Younger, J., Kuter, I., Bunnell, C., Rue, M., Gelman, R. and Winer, E., 2003. Central nervous system metastases in women who receive trastuzumab-based therapy for metastatic breast carcinoma. Cancer, 97(12), pp.2972-2977.

3. Tsukada, Y., Fouad, A., Pickren, J.W. and Lane, W.W., 1983. Central nervous system metastasis from breast carcinoma autopsy study. Cancer, 52(12), pp.2349-2354.

4. Iwamoto, T., Niikura, N., Ogiya, R., Yasojima, H., Watanabe, K.I., Kanbayashi, C., Tsuneizumi, M., Matsui, A., Fujisawa, T., Iwasa, T. and Shien, T., 2019. Distinct gene expression profiles between primary breast cancers and brain metastases from pair-matched samples. Scientific reports, 9(1), pp. $1-8$.

5. Salhia, B., Kiefer, J., Ross, J.T., Metapally, R., Martinez, R.A., Johnson, K.N., DiPerna, D.M., Paquette, K.M., Jung, S., Nasser, S. and Wallstrom, G., 2014. Integrated genomic and epigenomic analysis of breast cancer brain metastasis. PloS one, 9(1), p.e85448.

6. Zamolodchikov, D., Bai, Y., Tang, Y., McWhirter, J.R., Macdonald, L.E. and Alessandri-Haber, N., 2019. A Short Isoform of Coagulation Factor XII mRNA Is Expressed by Neurons in the Human Brain. Neuroscience, 413, pp.294-307.

7. Kurosawa, S. and Stearns-Kurosawa, D.J., 2014. Complement, thrombotic microangiopathy and disseminated intravascular coagulation. Journal of Intensive Care, 2(1), pp.1-8.

8. Dahlbäck, B., 2000. Blood coagulation. The Lancet, 355(9215), pp.1627-1632.

9. Renné, T., Pozgajová, M., Grüner, S., Schuh, K., Pauer, H.U., Burfeind, P., Gailani, D. and Nieswandt, B., 2005. Defective thrombus formation in mice lacking coagulation factor XII. The Journal of experimental medicine, 202(2), pp.271-281.

10.Renné, T. and Stavrou, E.X., 2019. Roles of factor XII in innate immunity. Frontiers in Immunology, 10, p.2011.

11.Wang, X., Wang, E., Kavanagh, J.J. and Freedman, R.S., 2005. Ovarian cancer, the coagulation pathway, and inflammation. Journal of translational medicine, 3(1), p.25.

12.McCulloch, P. and George, W.D., 1988. Promotion of metastasis by a specific complex of coagulation factors may be independent of fibrin formation. British journal of cancer, 58(2), pp.158-162.

PAGE 4 
PAGE 5 


\begin{tabular}{|l|l|l|l|l|l|l|}
\hline Rank & ID & p-value & t & B & Gene & Gene name \\
\hline 7919 & A_23_P167674 & $1.75 E-03$ & 3.3191208 & -1.751466 & F12 & $\begin{array}{l}\text { coagulation factor } \\
\text { XII }\end{array}$ \\
\hline
\end{tabular}

Table 2: F12 is differentially expressed in brain metastases in brain metastatic breast cancer.

Rank of differential expression, probe ID, $p$-value with respect to differential expression, $\mathrm{t}$, a moderated $\mathrm{t}$ statistic, B, the log-odds of differential expression between the two groups compared, gene and gene name are listed in this chart.

PAGE 6 


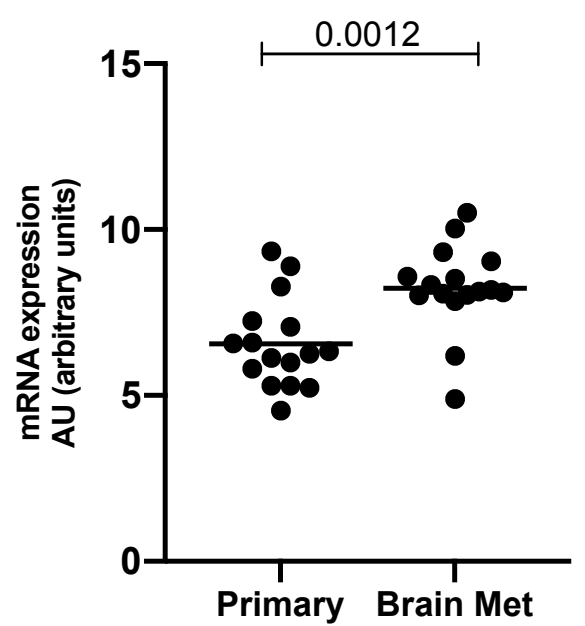

Figure 1: F12 is expressed at significantly higher levels in the brain metastases of patients with brain metastatic breast cancer.

The mRNA expression level of F12 in primary tumors of the breast (left) and in the brain metastases of brain metastatic breast cancer patients (right) is graphically represented here with mean F12 levels marked and the result of a statistical test evaluating difference in mRNA expression level between brain metastases and primary tumors of the breast, a $p$-value, listed above. 